\title{
Canonical double covers of circulants
}

\author{
Blas Fernandez ${ }^{a}$ and Ademir Hujdurović ${ }^{a, b}$ \\ ${ }^{a}$ University of Primorska, UP IAM, Muzejski trg 2, 6000 Koper, Slovenia \\ ${ }^{b}$ University of Primorska, UP FAMNIT, Glagoljaška 8, 6000 Koper, Slovenia
}

\begin{abstract}
The canonical double cover $B(X)$ of a graph $X$ is the direct product of $X$ and $K_{2}$. If $\operatorname{Aut}(B(X)) \cong \operatorname{Aut}(X) \times \mathbb{Z}_{2}$ then $X$ is called stable; otherwise $X$ is called unstable. An unstable graph is nontrivially unstable if it is connected, non-bipartite and distinct vertices have different neighborhoods. Circulant is a Cayley graph on a cyclic group. Qin et al. conjectured in [J. Combin. Theory Ser. B 136 (2019), 154-169] that there are no nontrivialy unstable circulants of odd order. In this paper we prove this conjecture.
\end{abstract}

Keywords: circulant, canonical double cover, unstable graph, automorphism group AMS Subject Classification (2010): 05C25, 05C76

\section{Introduction}

All groups considered in this paper are finite and all graphs are finite, simple and undirected. For a graph $X$, we denote by $V(X), E(X)$ and $\operatorname{Aut}(X)$ the vertex set, the edge set and the automorphism group of $X$, respectively. The canonical double cover (also called the bipartite double cover or the Kronecker cover) of a graph $X$, denoted by $B(X)$, is the direct product $X \times K_{2}$ (where $K_{2}$ denotes the complete graph on two vertices). This means that $V(B(X))=V(X) \times \mathbb{Z}_{2}$ and $E(B(X))=\{\{(x, 0),(y, 1)\} \mid\{x, y\} \in E(X)\}$. Canonical double covers have been studied by several authors, see for example [3, 10, 16, 25, 27]. It is well-known that $B(X)$ is connected if and only if $X$ is connected and non-bipartite, see [5. It can also be easily seen that $\operatorname{Aut}(B(X))$ contains a subgroup isomorphic to $\operatorname{Aut}(X) \times \mathbb{Z}_{2}$. However, determining the full automorphism group of $B(X)$ is not as trivial. Hammack and Imrich [4] investigated vertex-transitivity of direct product of graphs, and proved that for a non-bipartite graph $X$ and a bipartite graph $Y$, direct product $X \times Y$ is vertex-transitive if and only if both $B(X)$ and $Y$ are vertex-transitive. Hence, the problem of vertex-transitivity of direct product of graphs reduces to the problem of vertextransitivity of canonical double covers. If $\operatorname{Aut}(B(X))$ is isomorphic to $\operatorname{Aut}(X) \times \mathbb{Z}_{2}$ then the graph $X$ is called stable, otherwise it is called unstable. This concept was first defined by Marušič et al. [14] and studied later most notably by Surowski [22, 23], Wilson [26], Lauri et al. [11] and Qin et al. [19. A graph is said to be irreducible (or vertex-determining) if distinct vertices have different neighbours, and reducible otherwise. It is easy to see that the following graphs are all unstable: disconnected graphs, bipartite graphs with non-trivial automorphism group, and reducible graphs. An unstable graph is said to be nontrivialy unstable if it is non-bipartite, connected, and irreducible.

For a group $G$ and an inverse closed subset $S \subseteq G \backslash\left\{1_{G}\right\}$, the Cayley graph $\operatorname{Cay}(G, S)$ on $G$ with connection set $S$ is defined as the graph with vertex set $G$, with two vertices $x, y \in G$ being adjacent if and only if $x^{-1} y \in S$. A Cayley graph on a cyclic group is called circulant. Sabidussi [21] proved that a graph $X$ is a Cayley graph on a group $G$ if and only if $\operatorname{Aut}(X)$ has a regular subgroup isomorphic to $G$. It is easy to see that if $X$ is a Cayley graph on a group $G$, 
then its canonical double cover $B(X)$ is a Cayley graph on $G \times \mathbb{Z}_{2}$. The converse is not true in general, that is $B(X)$ can be a Cayley graph even if the graph $X$ is not Cayley. The problem of characterizing graphs with Cayley canonical double covers was studied by Marušič, Scapellato and Zagaglia Salvi [15], who introduced the class of generalized Cayley graphs (see also [7, 8]) and proved that if the canonical double cover of a generalized Cayley graph $\mathrm{X}$ is a stable graph, then $\mathrm{X}$ is a Cayley graph. The characterization of graphs whose canonical double covers are Cayley graphs was given by the second author in [6].

The problem of characterization of vertex-transitive unstable graphs was posed in 2, Problem 5.7]. However, the problem is difficult even when restricted to the class of circulant graphs. The problem of classification of nontrivially unstable circulants was posed in [19, Problem 1.2]. Qin et al proved that every circulant of odd prime order is stable (see [19, Theorem 1.4], and posed the following conjecture.

Conjecture 1.1. [19, Conjecture 1.3] There is no nontrivially unstable circulant of odd order.

In this paper we prove Conjecture 1.1, that is we prove the following theorem.

Theorem 1.2. Let $X$ be a connected irreducible circulant of odd order and let $B(X)$ be its canonical double cover. Then $\operatorname{Aut}(B(X)) \cong \operatorname{Aut}(X) \times \mathbb{Z}_{2}$.

Remark 1.3. Using Theorem 1.2, the automorphism group of a canonical double cover of any circulant $X$ of odd order can be determined in terms of the autormorphism group of $X$. Namely, if $X$ is a connected reducible circulant, then there exist a positive integer $d>1$ and a connected irreducible circulant $Y$, such that $X \cong Y>\overline{K_{d}}$. It is now easy to see that $B(X) \cong\left(B(Y)>\overline{K_{d}}\right)$. Using the result of Sabidussi [20] on the automorphism group of wreath product of graphs, we obtain $\operatorname{Aut}(B(X)) \cong \operatorname{Aut}\left(B(Y)<S_{d} \cong\left(\operatorname{Aut}(Y) \times \mathbb{Z}_{2}\right)<S_{d}\right.$.

\section{Preliminaries}

Following [18], an automorphism $\gamma$ of a bipartite graph $Y$ is called a strongly switching involution if $\gamma$ is an involution that swaps the two colour classes and fixes no edge. For a graph $X$ and a partition $\mathcal{P}$ of $V(X)$, the quotient graph of $X$ with respect to $\mathcal{P}$ is the graph whose vertex set is $\mathcal{P}$ with $B_{1}, B_{2} \in \mathcal{P}$ being adjacent if and only if there exist $x \in B_{1}$ and $y \in B_{2}$ such that $\{x, y\} \in E(X)$. The following result is proved in [18, Proposition 2.4].

Lemma 2.1. A bipartite graph $Y$ is isomorphic to the canonical double cover of a graph $X$ if and only if $\operatorname{Aut}(Y)$ contains a strongly switching involution $\gamma$ such that $X$ is isomorphic to the quotient graph of $Y$ with respect to the orbits of $\langle\gamma\rangle$.

The following lemma gives a necessary and sufficient condition for the stability of a graph, and it has been proved in [6, Corollary 2.5]. However, we give the proof here for the sake of completeness.

Lemma 2.2. Let $X$ be a connected non-bipartite graph, and let $B(X)$ be its canonical double cover. Let $\tau$ be the automorphism of $B(X)$ defined by $\tau(x, i)=(x, i+1)$. Then $X$ is stable if and only if $\tau$ is central in $\operatorname{Aut}(B(X)$.

Proof. Suppose first that $X$ is stable. Then $\operatorname{Aut}(B(X)) \cong \operatorname{Aut}(X) \times\langle\tau\rangle$, hence $\tau$ is central in $\operatorname{Aut}(B(X))$. Suppose now that $\tau$ is central in $\operatorname{Aut}(B(X))$. Let $\alpha \in \operatorname{Aut}(B(X))$ be arbitrary. 
Suppose first that $\alpha$ fixes the color classes of $B(X)$. Let $g$ be the permutation of $V(X)$ such that $\alpha(x, 0)=(g(x), 0)$. Since $\tau$ commutes with $\alpha$ it follows that $\alpha(x, 1)=\alpha(\tau(x, 0))=\tau(\alpha(x, 0))=$ $(g(x), 1)$. Let $\{x, y\} \in E(X)$. Then $\{(x, 0),(y, 1)\} \in E(B(X))$, and since $\alpha \in \operatorname{Aut}(B(X))$ it follows that $\{(g(x), 0),(g(y), 1)\} \in E(B(X))$. By definition of the canonical double cover, it follows that $\{g(x), g(y)\} \in E(X)$. It follows that $g \in \operatorname{Aut}(X)$. Hence, $\alpha$ is induced by the automorphism of $X$, that is $\alpha \in \operatorname{Aut}(X) \times \mathbb{Z}_{2}$. If $\alpha$ permutes the color classes of $B(X)$, then we apply the above arguments for $\alpha \tau$ which fixes the color classes, and conclude that $\alpha \tau \in \operatorname{Aut}(X) \times \mathbb{Z}_{2}$, hence $\alpha \in \operatorname{Aut}(X) \times \mathbb{Z}_{2}$. This finishes the proof.

Suppose that $X=\operatorname{Cay}\left(\mathbb{Z}_{k}, S\right)$ is a circulant of odd order $k$. Then it is straightforward to verify that $B(X)$ is a Cayley graph on $\mathbb{Z}_{k} \times \mathbb{Z}_{2}$, with connection set $S \times\{1\}$. Since the map $\alpha: \mathbb{Z}_{k} \times \mathbb{Z}_{2} \rightarrow \mathbb{Z}_{2 k}$ defined by $\alpha(x, i)=2 x+k i$ is an isomorphism, it follows that $B(X) \cong$ Cay $\left(\mathbb{Z}_{2 k}, k+2 S\right.$ ) (where elements of $S$ are now considered as elements of $\mathbb{Z}_{2 k}$ ), hence $B(X)$ is a circulant graph of order $2 k$. Observe that the mapping $k_{L}: \mathbb{Z}_{2 k} \rightarrow \mathbb{Z}_{2 k}$ defined by $k_{L}(x)=x+k$ is the automorphism of $B(X)$ that corresponds to the map $\tau$ from Lemma 2.2. Hence, for circulants of odd order we have the following result.

Lemma 2.3. Let $X=\operatorname{Cay}\left(\mathbb{Z}_{k}, S\right)$ be a connected circulant of odd order $k$. Then $X$ is stable if and only if the permutation $k_{L}$ is central in the automorphism group of Cay $\left(\mathbb{Z}_{2 k}, k+2 S\right)=B(X)$.

We will now give another characterization of stable vertex-transitive graphs.

Lemma 2.4. Let $X$ be a connected non-bipartite vertex-transitive graph. Let $B(X)$ be the canonical double cover of $X$, and let $A=\operatorname{Aut}(B(X))$. Then $X$ is stable if and only if $A_{(v, 0)}=A_{(v, 1)}$ for some $v \in V(X)$.

Proof. Suppose first that $X$ is stable. Then by Lemma 2.2 it follows that the map $\tau$ is central in $A$. If $\varphi \in A_{(v, 0)}$ then we have $\varphi(v, 1)=\varphi(\tau(v, 0))=\tau(\varphi(v, 0))=(v, 1)$. This shows that $A_{(v, 0)} \leq A_{(v, 1)}$. Since $B(X)$ is vertex-transitive, all stabilizers are of the same order, hence $A_{(v, 0)}=A_{(v, 1)}$.

Suppose now that $A_{(v, 0)}=A_{(v, 1)}$ for some $v \in V(X)$. Let $w \in V(X)$ be arbitrary, let $g$ be an automorphism of $X$ that maps $v$ into $w$, and let $\beta$ be the automorphism of $B(X)$ defined by $\beta(x, i)=(g(x), i)$. Then $A_{(w, 0)}=\beta A_{(v, 0)} \beta^{-1}=\beta A_{(v, 1)} \beta^{-1}=A_{(w, 1)}$.

Let $\alpha$ be an automorphism of $B(X)$. We will prove that $\alpha$ and $\tau$ commute. Without loss of generality, we may assume that $\alpha$ fixes the color classes of $B(X)$. Suppose that $\alpha(w, 0)=(u, 0)$, for some $u \in V(X)$. Let $h$ be an automorphism of $X$ that maps $u$ to $w$, and let $\gamma$ be the automorphism of $B(X)$ defined by $\gamma(x, i)=(h(x), i)$. Observe that $\gamma \alpha \in A_{(w, 0)}=A_{(w, 1)}$, hence $\gamma(\alpha(w, 1))=(w, 1)$. It follows that $\alpha(w, 1)=\gamma^{-1}(w, 1)=(u, 1)$. It is now straightforward to conclude that $\alpha \tau(w, 0)=\tau \alpha(w, 0)$. Since $w \in V(X)$ was arbitrary, it follows that $\alpha$ and $\tau$ commute. We conclude that $\tau$ is central in $\operatorname{Aut}(B(X))$. By Lemma 2.2 it follows that $X$ is stable.

For circulants, the above lemma implies the following.

Lemma 2.5. Let $X$ be a connected circulant of odd order $k$, let $B(X)$ be its canonical double cover and $A=A$ ut $(B(X))$. Then $X$ is stable if and only if $A_{0}=A_{k}$.

A Cayley graph $\Gamma=\operatorname{Cay}(G, S)$ is said to be normal if $G_{L}$ is a normal subgroup of $\operatorname{Aut}(\Gamma)$, or equivalently if $\operatorname{Aut}(\Gamma)_{0}=\operatorname{Aut}(G, S)$, where $\operatorname{Aut}(G, S)=\{\varphi \in \operatorname{Aut}(G) \mid \varphi(S)=S\}$. 
Lemma 2.6. Let $G$ be an abelian group of odd order, and let $X=C a y(G, S)$ be a connected Cayley graph on $G$. If $B(X)=C a y\left(G \times \mathbb{Z}_{2}, S^{\prime}\right)$ is a normal Cayley graph then $X$ is stable.

Proof. Since $B(X)$ is normal Cayley graph, it follows that each element of $\operatorname{Aut}(B(X))$ is a composition of some element of $\left(G \times \mathbb{Z}_{2}\right)_{L}$ with some element of $\operatorname{Aut}\left(G \times \mathbb{Z}_{2}\right)$. Let $\tau$ be the automorphism of $B(X)$ defined by $\tau(x, i)=(x, i+1)$. Let $t$ be the unique element of order 2 in $G \times \mathbb{Z}_{2}$. Observe that $\tau=t_{L}$. Since $G$ is abelian, it follows that each element of $\left(G \times \mathbb{Z}_{2}\right)_{L}$ commutes with $\tau$. Let $\varphi \in \operatorname{Aut}\left(G \times \mathbb{Z}_{2}\right)$. Then $\varphi(t)=t$, since group automorphisms preserve the order of elements, and $t$ is the unique element of order 2 in $G \times \mathbb{Z}_{2}$. It follows that $\left(\varphi t_{L}\right)(x)=\varphi(t x)=t \varphi(x)=\left(t_{L} \varphi\right)(x)$. This shows that $\tau=t_{L}$ commutes with every element of $\operatorname{Aut}(G)$. The result now follows by Lemma 2.3 ,

The following lemma tells that a normal circulant of even order not divisible by four has a unique regular cyclic subgroup.

Lemma 2.7. 24, Theorem 5.2.2] Let $k$ be an odd positive integer, and let $X=C a y\left(\mathbb{Z}_{2 k}, S\right)$. Let $A=A u t(X)$ admitting a normal cyclic regular subgroup $H$. Then $H$ is the unique regular cyclic subgroup contained in A.

The wreath (lexicographic) product $\Sigma \imath \Gamma$ of a graph $\Gamma$ by a graph $\Sigma$ is the graph with vertex set $V(\Sigma) \times V(\Gamma)$ such that $\left\{\left(u_{1}, u_{2}\right),\left(v_{1}, v_{2}\right)\right\}$ is an edge if and only if either $\left\{u_{1}, v_{1}\right\} \in E(\Sigma)$, or $u_{1}=v_{1}$ and $\left\{u_{2}, v_{2}\right\} \in E(\Gamma)$. Observe that $\Sigma \imath \Gamma$ is the graph obtained by substituting a copy of $\Gamma$ for each vertex of $\Gamma$.

The deleted wreath (deleted lexicographic) product of a graph $\Sigma$ and $\overline{K_{d}}$, denoted by $\Sigma \imath_{d} \overline{K_{d}}$, is the graph with vertex set $V(\Sigma) \times \mathbb{Z}_{d}$, such that $\left\{\left(u_{1}, i\right),\left(v_{1}, j\right)\right\}$ is an edge if and only if $\left\{u_{1}, v_{1}\right\} \in$ $E(\Sigma)$ and $i \neq j$. Observe that $\Sigma \imath_{d} \overline{K_{d}}$ can be obtained from $\Sigma \imath \overline{K_{d}}$ by removing $d$ disjoint copies of $\Sigma$. Observe that the canonical double cover of a graph $X$ is isomorphic to $X \imath_{d} \overline{K_{2}}$ (see [19, Example 2.1]). The following result gives a characterization of all arc-transitive circulants.

Lemma 2.8. 9, 12 Let $\Gamma$ be a connected arc-transitive circulant of order $n$. Then one of the following holds:

(i) $\Gamma \cong K_{n}$;

(ii) $\Gamma=\Sigma \imath \overline{K_{d}}$, where $n=m d, m, d>1$ and $\Sigma$ is a connected arc-transitive circulant of order $m$;

(iii) $\Gamma=\Sigma \iota_{d} \overline{K_{d}}$ where $n=m d, d>3, \operatorname{gcd}(d, m)=1$ and $\Sigma$ is a connected arc-transitive circulant of order $m$;

(iv) $\Gamma$ is a normal circulant.

The following result is a direct consequence of [2, Theorem 5.3].

Lemma 2.9. Let $d \geq 3$ be an integer, let $\Sigma$ be an irreducible vertex-transitive graph whose order is not divisible by $d$, and let $\Gamma=\Sigma \imath_{d} \overline{K_{d}}$. Then $\operatorname{Aut}(\Gamma) \cong \operatorname{Aut}(\Sigma) \times S_{d}$.

Proof. Since $\Sigma$ is vertex-transitive, and $\operatorname{Aut}(\Gamma)$ contains a subgroup isomorphic to $\operatorname{Aut}(\Sigma) \times S_{d}$, it follows that $\Gamma$ is vertex-transitive. Since $\Sigma$ is reducible, by [2, Theorem 5.3] it follows that $\operatorname{Aut}(\Gamma) \neq \operatorname{Aut}(\Sigma) \times S_{d}$ if and only if the conditions $(i)-(i i i)$ of [2, Theorem 5.3] hold. However, since $|V(\Sigma)|$ is not divisible by $d$, it follows that condition (ii) of [2, Theorem 5.3] doesn't hold. We conclude that $\operatorname{Aut}(\Gamma) \cong \operatorname{Aut}(\Sigma) \times S_{d}$. 
Lemma 2.10. [19, Lemma 2.3] The canonical double cover $B(X)$ of a graph $X$ is reducible if and only if $X$ is reducible.

The following result can be extracted from the proof of [17, Proposition 2.1]. However, as the statement of [17, Proposition 2.1] is a bit weaker, we include the proof here for the sake of completeness.

Lemma 2.11. Let $X=\operatorname{Cay}(G, S), A=A u t(X)$ and let $K \subset S$ such that $\varphi(K)=K$ for every $\varphi \in A_{1}$. Then $\varphi(\langle K\rangle)=\langle K\rangle$ for every $\varphi \in A_{1}$. Moreover, if $K$ is inverse closed, then $\varphi$ induces an automorphism of $\operatorname{Cay}(\langle K\rangle, K)$.

Proof. Let $x \in G$ and $\omega \in A_{x}$ be arbitrary, and let $M$ be any subset of $G$ such that $\varphi(M)=M$ for every $\varphi \in A_{1}$. Observe that $\left(x_{L}\right)^{-1} \omega x_{L} \in A_{1}$. By the assumption on $A_{1}$ and $M$ it follows that $\left(x_{L}\right)^{-1} \omega x_{L}(M)=M$. Therefore, $\omega(x M)=x M$ for every $x \in G$ and every $\omega \in A_{x}$.

We claim that $\varphi\left(K^{t}\right)=K^{t}$ for every positive integer $t$. The proof is by induction on $t$. If $t=1$, the claim follows from the hypothesis. Suppose that $\varphi\left(K^{t}\right)=K^{t}$. Let $\varphi \in A_{1}$ and let $k_{1} \in K$ be arbitrary. Observe that $\left(k_{1} k_{2}^{-1}\right)_{L} \varphi \in A_{k_{1}}$. Applying the first part of the proof with $M=K^{t}$ and $x=k_{1}$, it follows that $\left(k_{1} k_{2}^{-1}\right)_{L} \varphi\left(k_{1} K^{t}\right)=k_{1} K^{t}$. This implies that $\varphi\left(k_{1} K^{t}\right)=$ $k_{2} K^{t}=\varphi\left(k_{1}\right) K^{t} \subseteq K^{t+1}$. Since this is true for every $k_{1} \in K$, it follows that $\varphi\left(K^{t+1}\right)=K^{t+1}$.

Since $\langle K\rangle=K \cup K^{2} \cup \ldots \cup K^{t}$ for some positive integer $t$, it follows that $\varphi(\langle K\rangle)=\langle K\rangle$. Moreover, let $e$ be an arbitrary edge of $\operatorname{Cay}(\langle K\rangle, K)$. Then $e=\left\{k_{1}, k_{1} k_{2}\right\}$ for some $k_{1}, k_{2} \in K$. As we proved that $\varphi\left(k_{1} K^{t}\right)=\varphi\left(k_{1}\right) K^{t}$ for every positive integer $t$, it follows that $\varphi\left(k_{1} K\right)=\varphi\left(k_{1}\right) K$. Since $\varphi\left(k_{1} k_{2}\right) \in \varphi\left(k_{1} K\right)=\varphi\left(k_{1}\right) K$ we conclude that $\varphi\left(k_{1} k_{2}\right)=\varphi\left(k_{1}\right) k$ for some $k \in K$. This shows that $\varphi(e)$ is an edge of $\operatorname{Cay}(\langle K\rangle, K)$, hence $\varphi$ induces an automorphism of $\operatorname{Cay}(\langle K\rangle, K)$.

\section{Main result}

The following lemma gives a partial generalization of [19, Theorem 1.6], where it is proved that there is no arc-transitive nontrivally unstable circulant. It is easy to see that $B(X)$ is arc-transitive if $X$ is arc-transitive.

Lemma 3.1. Let $X$ be a nontrivialy unstable circulant of odd order $m$. Then $B(X)$ is not arctransitive.

Proof. Suppose that $B(X)$ is an arc-transitive circulant. Since $B(X)$ is also a connected circulant, by Lemma 2.8, it follows that $B(X)$ is a complete graph, normal circulant, wreath product or a deleted wreath product. If $B(X)$ is a complete graph, then it must be isomorphic to $K_{1}$ or $K_{2}$, since they are the only bipartite complete graphs. However, it is easy to see that none of them is a canonical double cover of some graph. Lemma 2.6 implies that $B(X)$ is not a normal circulant. If $B(X)$ is a wreath product, then $B(X)$ is reducible, hence by Lemma 2.10 it follows that $X$ is also reducible, contrary to the assumption that $X$ is nontrvialy unstable. Therefore, we may assume that $B(X)$ is the deleted wreath product. Suppose that $B(X) \cong \Sigma \imath_{d} \overline{K_{d}}$, where $2 m=t d, d>3$, $\operatorname{gcd}(d, t)=1$ and $\Sigma$ is a connected arc-transitive circulant of order $t$. Since $B(X)$ is bipartite and $d>3$ it follows that $\Sigma$ is also bipartite, hence order of $\Sigma$ is even.

If $\Sigma$ is reducible, then $\Sigma \cong \Sigma_{1} 2 \overline{K_{d_{1}}}$, where $\Sigma_{1}$ is an irreducible circulant. Then by [2, Proposition 4.5], it follows that $B(X) \cong \Sigma \imath_{d} \overline{K_{d}} \cong\left(\Sigma_{1} \imath_{d} \overline{K_{d}}\right)<\overline{K_{d_{1}}}$, implying that $B(X)$ is reducible. By Lemma 2.10 it follows that $X$ is also reducible, a contradiction.

Suppose now that $\Sigma$ is irreducible. Then by Lemma 2.9 it follows that $\operatorname{Aut}(B(X)) \cong \operatorname{Aut}(\Sigma) \times$ $S_{d}$. Recall that $\Sigma$ is a connected arc-transitive bipartite circulant of even order. If $\Sigma$ is a wreath 
product, then again $B(X)$ is a wreath product, hence $B(X)$ is reducible, and consequently also $X$ is reducible, contrary to the assumption that $X$ is nontrivialy unstable. We conclude that $\Sigma$ is a normal circulant, or $\Sigma \cong \Sigma_{1} \imath_{d} \overline{K_{d_{1}}}$, where $\Sigma_{1}$ is an arc-transitive circulant. Applying the same arguments for $\Sigma_{1}$, we conclude that $\Sigma_{1}$ is a normal circulant, or a deleted wreath product. As $\Gamma$ is finite, this process has to terminate, so eventually we will get $B(X)=\left(\Sigma_{t} \imath_{d} \overline{K_{d_{t}}}\right) \imath_{d} \ldots \imath_{d} \overline{K_{d}}$, where $\Sigma_{t}$ is a normal circulant of even order and $\operatorname{Aut}(B(X)) \cong \operatorname{Aut}\left(\Sigma_{t}\right) \times S_{d_{t}} \times \ldots \times S_{d}$.

Observe that $S_{d_{t}} \times \ldots \times S_{d}$ is a normal subgroup of $\operatorname{Aut}(B(X))$, hence its orbits form a system of imprimitivity for $\operatorname{Aut}(B(X))$. Let $\mathcal{P}$ denote the set of orbits of $S_{d_{t}} \times \ldots \times S_{d}$ on $V(B(X))$. Observe that the quotient graph of $B(X)$ with respect to $\mathcal{P}$ is isomorphic to $\Sigma_{t}$. The group $\left(\mathbb{Z}_{2 m}\right)_{L}$ projects into cyclic regular subgroup of $\operatorname{Aut}\left(\Sigma_{t}\right)$. For $g \in \operatorname{Aut}(B(X))$, let $g / \mathcal{P}$ denote the permutation induced by the action of $g$ on $\mathcal{P}$. Since $\Sigma_{t}$ is a normal circulant, by Lemma 2.7 it follows that $\left(\mathbb{Z}_{2 m}\right)_{L} / \mathcal{P}$ is a normal cyclic regular subgroup of $\operatorname{Aut}\left(\Sigma_{t}\right)$, hence $m_{L} / \mathcal{P}$ is central in $\operatorname{Aut}\left(\Sigma_{t}\right)$. If follows that $m_{L}$ is central in $\operatorname{Aut}\left(\Sigma_{t}\right) \times S_{d_{t}} \times \ldots \times S_{d}$. The result now follows by Lemma 2.3 ,

In the following lemma, we consider graphs that can be realised as a canonical double cover of some connected arc-transitive circulant of odd order, and derive certain important properties of their automorphism groups.

Lemma 3.2. Let $m$ be an odd positive integer, and let $\Gamma$ be a connected bipartite arc-transitive circulant of order $2 m$ and even valency, and let $A=A u t(\Gamma)$. Then one of the following holds:

(i) $A_{0}=A_{m}$, or

(ii) $\Gamma \cong \Gamma_{1} 2 \overline{K_{d}}$ where $\Gamma_{1}$ is an irreducible arc-transitive circulant of even order $2 m_{1}$ and $\operatorname{Aut}\left(\Gamma_{1}\right)_{0}=\operatorname{Aut}\left(\Gamma_{1}\right)_{m_{1}}$.

Proof. Since $\Gamma$ is a circulant of order $2 m$ and has even valency, it follows that $\Gamma \cong \operatorname{Cay}\left(\mathbb{Z}_{2 m}, S\right)$ where $S$ does not contain element $m$. This implies that the automorphism $m_{L}: x \mapsto m+x$ is a strongly switching involution of $\Gamma$. By Lemma 2.1, it follows that $\Gamma$ is the canonical double cover of the graph $X$ obtained as the quotient graph of $\Gamma$ with respect to the orbits of $m_{L}$. Observe that $X$ is a connected and non-bipartite circulant of order $m$, since $\Gamma$ is a connected circulant of order $2 m$.

If $X$ is a stable graph, then by Lemma 2.5 it follows that $\Gamma$ satisfies condition $(i)$, and we are done. As $\Gamma$ is arc-transitive, by Lemma 3.1 it follows that $X$ is not nontrivialy unstable. Therefore, $X$ is trivially unstable, and since it is connected and non-bipartite, it follows that $X$ is reducible. We conclude that $X \cong \Sigma \imath \overline{K_{d}}$, where $\Sigma$ is a connected irreducible circulant of order $m_{1}$, with $m_{1}$ being odd.

Observe that $\Gamma \cong X \times K_{2} \cong\left(\Sigma \imath \overline{K_{d}}\right) \times K_{2} \cong\left(\Sigma \times K_{2}\right) \imath \overline{K_{d}}$. Let $\Gamma_{1}=\Sigma \times K_{2}$. Since $\Gamma$ is an arc-transitive circulant, it follows that $\Gamma_{1}$ is an arc-transitive circulant (see [13, Remark 1.2]). Since $\Gamma_{1}$ is a canonical double cover of $\Sigma$, by Lemma 3.1 it follows that $\Sigma$ is not non-trivially unstable. Recall that $\Sigma$ is irreducible, connected and non-bipartite. We conclude that $\Sigma$ is stable, hence by Lemma 2.5 it follows that $\operatorname{Aut}\left(\Gamma_{1}\right)_{0}=\operatorname{Aut}\left(\Gamma_{1}\right)_{m_{1}}$.

We are now ready to prove the main result of this paper. We will show that there is no non-trivially unstable circulant of odd order.

Theorem 1.2 (restated). Let $X$ be a connected irreducible circulant of odd order and let $B(X)$ be its canonical double cover. Then $\operatorname{Aut}(B(X)) \cong \operatorname{Aut}(X) \times \mathbb{Z}_{2}$. 
Proof. Let $X=\operatorname{Cay}\left(\mathbb{Z}_{m}, S\right)$ be a connected irreducible circulant of odd order $m$. If $X$ is stable, the result follows by the definition. It is clear that $X$ is non-bipartite, as it is of odd order. Hence, we may assume that $X$ is a nontrivially unstable. We have that $B(X)=\operatorname{Cay}\left(\mathbb{Z}_{2 m}, S^{\prime}\right)$ is a circulant of order $2 m$, where $S^{\prime}=m+2 S$. Let $A=\operatorname{Aut}(B(X))$. If $A_{0}$ is transitive on $S^{\prime}$, then $B(X)$ is arc-transitive, and the result follows by Lemma 3.1. Let $S_{1}, \ldots, S_{k}$ be the orbits of $A_{0}$ on $S^{\prime}$. Observe that $S_{i}=-S_{i}$ (since mapping $i: x \mapsto-x$ is contained in $A_{0}$ ), and $m \notin S_{i}$, for every $i \in\{1, \ldots, k\}$. Let $\Gamma_{i}=\operatorname{Cay}\left(\left\langle S_{i}\right\rangle, S_{i}\right)$. Observe that $\left\langle S_{i}\right\rangle$ is a subgroup of $\mathbb{Z}_{2 m}$ of even order, hence it contains the element of order 2 , that is $m \in\left\langle S_{i}\right\rangle$ for every $i \in\{1, \ldots, k\}$. By Lemma 2.11 every element of $A_{0}$ fixes $\Gamma_{i}$, hence it follows that every element of $A_{0}$ induces an automorphism of $\Gamma_{i}$. As $A_{0}$ acts transitively on $S_{i}$ it follows that $\Gamma_{i}$ is arc-transitive. Therefore, $\Gamma_{i}$ is an arc-transitive circulant of even order (not divisible by 4) and even valency. Hence we can apply Lemma 3.2 to each of the graphs $\Gamma_{i}$. If for some $i$ we have that $\Gamma_{i}$ satisfies condition 1 of Lemma 3.2, it follows that every automorphism of $B(X)$ that fixes 0 , must also fix $m$, hence $A_{0}=A_{m}$ and by Lemma 2.5 it follows that $X$ is stable, a contradiction.

We can now assume that $\Gamma_{i} \cong \Sigma_{i} \lambda \overline{K_{d_{i}}}$, where $\Sigma_{i}$ is an irreducible arc-transitive circulant of even order satisfying condition $(i)$ from Lemma 3.2. Let $R$ be the equivalence relation of "having the same neighbourhood" defined on $V\left(\Gamma_{i}\right)$. The equivalence classes of this relation are all of size $d_{i}$, and form a system of imprimitivity for $\operatorname{Aut}\left(\Gamma_{i}\right)$. Let $H_{i}$ be the kernel of the action of $\left\langle S_{i}\right\rangle_{L}$ (which is a regular cyclic subgroup of $\operatorname{Aut}\left(\Gamma_{i}\right)$ ) on the partition induced by $R$. The permutation group $\left\langle S_{i}\right\rangle_{L} / H_{i}$ induced by the action of $\left\langle S_{i}\right\rangle_{L}$ on the classes of $R$ is a cyclic regular group. Since $H_{i}$ is semiregular on $V\left(\Gamma_{i}\right)$, it follows that the equivalence classes of $R$ coincide with the orbits of $H_{i}$. It follows that $S_{i}$ is a union of cosets of the subgroup $H_{i}$ of $\left\langle S_{i}\right\rangle$. Observe that the element of order 2 in the quotient group $\left\langle S_{i}\right\rangle / H_{i}$ is $m+H_{i}$.

Recall that $\Sigma_{i}$ has the property that the stabilizer of the identity and the element of order 2 in the cyclic regular subgroup of $\operatorname{Aut}\left(\Sigma_{i}\right)$ are equal, which implies that every automorphism of $\Gamma_{i}$ that fixes 0 , fixes setwise coset $m+H_{i}$. As every automorphism of $\Gamma$ that fixes 0 induces automorphism of $\Gamma_{i}$, it follows that every automorphism of $\Gamma$ that fixes 0 fixes setwise $m+H_{i}$. If $G C D\left(d_{1}, \ldots, d_{k}\right)=d>1$, then $H=H_{1} \cap \ldots \cap H_{k}$ has order $d$. It follows that $S^{\prime}$ is a union of cosets of $H$, hence $B(X)$ is a wreath product with $\overline{K_{d}}$. This shows that $B(X)$ is reducible, and by Lemma 2.10 it follows that $X$ is also reducible, contrary to the assumption that $X$ is nontrivially unstable circulant.

If $G C D\left(d_{1}, \ldots, d_{k}\right)=1$, then it follows that $H_{1} \cap \ldots \cap H_{k}=\{0\}$. As observed above, every automorphism of $\Gamma$ fixes setwise each of the sets $m+H_{i}$, for $i \in\{1, \ldots, k\}$, hence it also fixes their intersection. Since $\left(m+H_{1}\right) \cap \ldots \cap\left(m+H_{k}\right)=\{m\}$, by Lemma 2.5 it follows that $X$ is stable, a contradiction. This finishes the proof.

For further research, we propose the following problem.

Problem 3.3. Does there exist a nontrivially unstable Cayley graph on an Abelian group of odd order.

\section{Acknowledgement}

This work has been supported by the Slovenian Research Agency (Young researchers program, research programs P1-0404 and P1-0285, and research projects J1-1691, J1-1694, J1-1695, J1-9110, N1-0102 and N1-0140). 


\section{References}

[1] S. Bhoumik, E. Dobson and Y. Morris, On the automorphism groups of almost all circulant graphs and digraphs, Ars Math. Contemp. 7 (2014), 487-506.

[2] T. Dobson, Š. Miklavič, and P. Šparl, On Automorphism Groups of Deleted Wreath Products. Mediterr. J. Math. 16, 149 (2019).

[3] Y.Q. Feng, K. Kutnar, A. Malnič and D. Marušič, On 2-fold covers of graphs, J. Combin. Theory Ser. B 98 (2008), 324-341.

[4] R. Hammack and W. Imrich, Vertex-transitive direct products of graphs, Electron. J. Combin. 25 (2018), Paper 2.10, 16 pp.

[5] R. Hammack, W. Imrich and S. Klavžar. Handbook of Product Graphs, Second Edition. Discrete Mathematics and Its Applications. Taylor \& Francis, 2011.

[6] A. Hujdurović, Graphs with Cayley canonical double covers. Discrete Math. 342 (2019), no. 9, 2542-2548.

[7] A. Hujdurović, K. Kutnar and D. Marušič, Vertex-transitive generalized Cayley graphs which are not Cayley graphs, European J. Combin. 46 (2015), 45-50.

[8] A. Hujdurović, K. Kutnar, P. Petecki and A. Tanana, On automorphisms and structural properties of generalized Cayley graphs, Filomat 31 (2017), 4033-4040.

[9] I. Kovács, Classifying arc-transitive circulants , J. Algebr. Combin. 20 (2004), 353-358.

[10] M. Krnc and T. Pisanski, Characterization of generalized Petersen graphs that are Kronecker covers, (2018), arXiv: 1802.07134 [math. CO]

[11] J. Lauri, R. Mizzi and R. Scapellato, Unstable graphs: a fresh outlook via TF-automorphisms, Ars Math. Contemp. 8 (2015), 115-131.

[12] C. H. Li, Permutation groups with a cyclic regular subgroup and arc transitive circulants, J. Algebraic Combin. 21 (2005), 131136.

[13] C. H. Li, D. Marušič, J. Morris, Classifying arc-transitive circulants of square-free order. J. Algebraic Combin. 14 (2001), no. 2, 145-151.

[14] D. Marušič, R. Scapellato and N. Zagaglia Salvi. A characterization of particular symmetric (0, 1)-matrices. Linear Algebra Appl. 119 (1989), 153-162.

[15] D. Marušič, R. Scapellato and N. Zagaglia Salvi, Generalized Cayley graphs, Discrete Math. 102 (1992), 279-285.

[16] R. Nedela and M. Škoviera, Regular embeddings of canonical double coverings of graphs, $J$. Combin. Theory Ser. B 67 (1996), 249-277.

[17] L.A. Nowitz, M.E. Watkins, Graphical regular representations of non-abelain groups, I, Canad. J. Math. 24 (1972) 994-1008. 
[18] W. Pacco and R. Scapellato, Digraphs having the same canonical double covering, Discrete Math. 173 (1997), 291-296.

[19] Y.L. Qin, B. Xia and S. Zhou, Stability of circulant graphs, https://arxiv.org/abs/1802.04921.

[20] G. Sabidussi, The composition of graphs, Duke Math J. 26 (1959), 693-696.

[21] G. Sabidussi, On a class of fixed-point-free graphs, Proc. Amer. Math. Soc. 9 (1958), 800-804.

[22] D. B. Surowski, Stability of arc-transitive graphs, J. Graph Theory, 38 (2001), 95-110.

[23] D. B. Surowski, Automorphism groups of certain unstable graphs. Math. Slovaca, 53 (2003), 215-232.

[24] Y. Xu (2018), Normal and non-normal cayley graphs, [Doctoral dissertation, The University of Western Australia], https://doi.org/10.26182/5c496c0ddd2da

[25] D. Waller, Double covers of graphs, Bull. Austral. Math. Soc. 14 (1976) 233-248.

[26] S. Wilson, Unexpected symmetries in unstable graphs, J. Combin. Theory Ser. B 98 (2008), 359-383.

[27] B. Zelinka, On double covers of graphs, Math. Slovaca 32 (1982) 49-54. 